\title{
Depression: From Sorrow to Melancholia
}

\author{
Gabriel Miranda Nava* \\ Chief of Neurology of the Hospital Center of the Presidential General Staff, Mexico
}

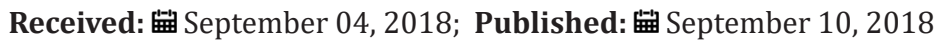

*Corresponding author: Gabriel Miranda Nava, Chief of Neurology of the Hospital Center of the Presidential General Staff, Mexico

\section{Opinion}

Sometimes we have felt so sad and so disappointed for a few hours or days, a situation that leads us to sleep badly, have constant awakenings, decreased appetite, easy crying, tiredness, attention deficit, cranky, even wanting to you "swallow the earth" which is a minor thought to "want to die"; These situations are normal in the mood swings we experience throughout the day or in a few weeks, and that is recovering over time, thanks to the adjustment in your brain experienced by neurotransmitters, which we have already mentioned are substances chemical in the brain that allow to carry out the different phases of thought. This series of symptoms or sensations come to us from a series of external stimuli or characteristic experiences that all, absolutely all of us must cross at some time in our life, as are the love breaks, both the conjugal separation and the diverse breaks of the courtship, the loss of a loved one, an economic bankruptcy, a robbery, a labor dismissal, in short. The big problem lies in the times, that is, when this series of situations are presented at the passage of a month, and that they become incapacitated for their work, for their family, for life in general, this is when it happens to be a moment of sadness at the moment of using the word high-sounding: depression. the highsounding word: depression.

And it is that the depression does not respect age, sex, religion, social position or another variant of the human being; It has different presentations since in children we can observe it with attention deficit, irritability, socially unsocial personality or that avoids meetings, or puncher or letting it hit at school; in adolescents with sleep difficulties, whether with lack or excess, sadness, easy crying, loss of daily habits; or in elderly people with severe forgetfulness that simulates Alzheimer's disease. In Mexico of every 100 people, 12 suffer only depression or its variants, without counting other types of mental illness, and of these 12 one will try to commit suicide in one way or another; These data do not mention the great loss of working days and that apart is the first cause of consultation at the level of the specialty of Psychiatry and the first ten causes of general consultation. That is why we are going to apply the following test, called Beck's, to know if we have depression or not and make the decision to attend a medical consultation.
Beck Depression Inventory.

I.

a. I do not feel sad

b. I feel sad.

c. I feel sad continuously and I can not stop being.

d. I feel so sad or so miserable that I can not stand it.

II.

a. I do not feel especially discouraged about the future.

b. I feel discouraged about the future.

c. I feel like I do not have to wait for anything.

d. I feel that the future is hope less and things will not improve.

III.

a. I do not feel unsuccessful.

b. I think I have failed more than most people.

c. When I look back, I only see failure after failure.

d. I feel a totally unsuccessful person.

IV.

a. Things satisfy me as much as before.

b. I do not enjoy things as much as before.

c. I no longer get an authentic satisfaction of things.

d. I am dissatisfied or bored with everything.

V.

a. I do not feel especially guilty.

b. I feel guilty on many occasions.

c. I feel guilty most of the time.

d. I feel guilty constantly. 
VI.

a. I do not think I'm being punished.

b. I feel like I'm going to be punished.

c. I hope to be punished.

d. I feel like I am being punished.

VII.

a. I am not disappointed in myself.

b. I am disappointed in myself.

c. I am ashamed of myself.

d. I detest myself

VIII.

a. I do not consider myself worse than anyone else.

b. I criticize myself for my weaknesses or for my mistakes.

c. I continually blame myself for my faults.

d. I blame myself for all the bad things that happen.

IX.

a. I do not have any thoughts of suicide.

b. Sometimes I think about committing suicide, but I would not commit it.

c. I would like to commit suicide.

d. I would kill myself if I had the chance.

$\mathrm{X}$.

a. I do not cry more than I used to cry.

b. Now I cry more than before.

c. or I cry continuously.

d. Before I was able to cry, but now I cannot, even if I want to. eleven.

XI.

a. I'm not more irritated than usual in me.

b. I annoy or irritate more easily than before.

c. I feel irritated continuously.

d. I am not absolutely irritated by the things that used to irritate me.

XII.

a. I have not lost interest in others.

b. I am less interested in others than before.

c. I have lost most of my interest in others. d. I have lost all interest in others.

XIII.

a. I make decisions more or less as I have always done.

b. I avoid making decisions more than before.

c. Making decisions is much more difficult than before.

d. It is already impossible for me to make decisions.

XIV.

a. I do not think I look worse than before.

b. I am afraid that now I look older or unattractive.

c. I think there have been permanent changes in my appearance that make me look unattractive.

d. I think I look horrible.

XV.

a. Work as before.

b. It takes me an extra effort to start doing something.

c. I have to force myself a lot to do something.

d. I can not do anything at all.

XVI.

a. I sleep as well as ever.

b. I do not sleep as well as before.

c. I wake up an hour or two earlier than usual and find it difficult to go back to sleep.

d. I wake up several hours earlier than usual and I can not go back to sleep.

XVII.

a. I do not feel more tired than usual.

b. I get tired more easily than before.

c. I get tired as soon as I do anything.

d. I am too tired to do anything.

XVIII.

a. My appetite has not diminished.

b. I do not have as good an appetite as before.

c. Now I have much less appetite.

d. I have completely lost my appetite.

XIX.

a. Lately I have lost little weight, or I have not lost anything.

b. I have lost more than 2 kilos and a half. 
c. I have lost more than 4 kilos.

d. I have lost more than 7 kilos.

e. I am on a diet to lose weight YES / NO.

XX.

a. I am not worried about my health more than usual.

b. I am worried about physical problems such as pain, discomfort, upset stomach or constipation.

c. I am worried about my physical problems and find it difficult to think about anything else.

d. I am so worried about my physical problems that I am unable to think of anything.

XXI.

a. I have not observed any recent changes in my interest.

b. I am less interested in sex than before. c. I am much less interested in sex.

d. I have totally lost my interest in sex.

\section{Guide for the Interpretation of the Beck Depression Inventory:}

Score Depression level*

a. 1-10: These highs and lows are considered normal.

b. 11-16: Mild disturbance of mood.

c. 17-20: Intermittent depression states.

d. 21-30: Moderate depression.

e. 31-40: Severe depression.

f. +40: Extreme depression.

*A persistent score of 17 or more indicates that you may need help professional.
Commons Attribution 4.0 License

To Submit Your Article Click Here: Submit Article

DOI: $10.32474 /$ SJPBS.2018.01.000106

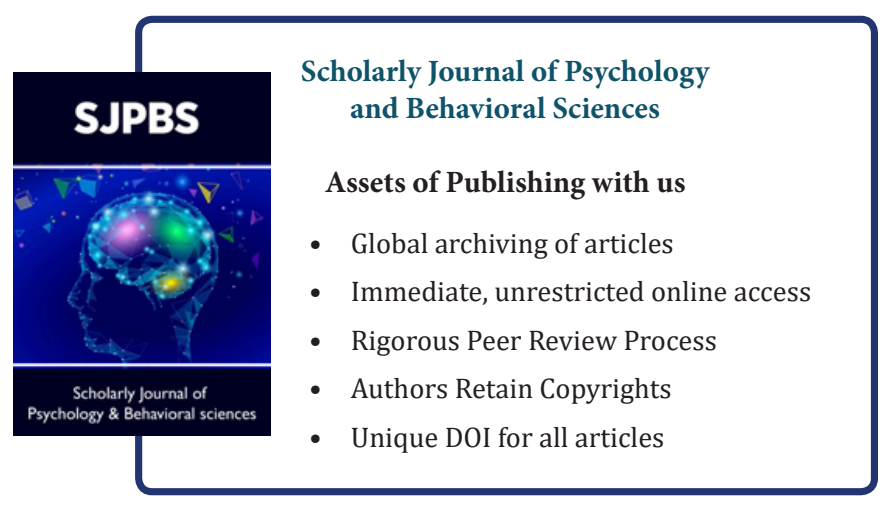

